\title{
Análisis de rendimiento y costo de los combustibles ecopaís y super
}

\section{Performance and cost analysis for ecopaís and super fuels}

Ing. Fredy Leonardo Morquecho Andrade, MSc. Universidad Internacional del Ecuador, Ecuador Autor para correspondencia: frmorquechoan@internacional.edu.ec Fecha de recepción: 15 de agosto de 2018 - Fecha de aceptación: 15 octubre de 2018

Resumen: Este estudio se basa en una investigación objetiva, sistemática y controlada con el propósito de analizar y examinar los resultados de rendimiento, economía y pruebas de emisiones entre la gasolina Súper y la gasolina Ecopaís, en un vehículo marca Chevrolet modelo Aveo del año 2017, motor 1500 cc, de cuatro cilindros. Se realiza un estudio comparativo en cuatro rutas preestablecidas, horarios de circulación y bajo similares condiciones de trabajo del vehículo. En la parte metodológica se revisó bibliografía relacionada con este tema para obtener datos correctos con respecto al rendimiento de los dos tipos de combustibles disponibles en el Ecuador, se utilizaron aplicaciones de apoyo disponibles en internet como el Google Maps, GPS velocímetro y Hudspeed. Usando estas herramientas digitales, como lo son los medidores de ruta se realizó las diferentes pruebas de rendimiento, para determinar si ambos tipos de combustible cumplen con los parámetros de consumo y niveles de contaminación del vehículo. De esta forma demostrar si en realidad se reducen los costos de consumo de combustible y mantenimientos a largo plazo de un vehículo con motor de combustión interna. Los resultados que se muestran en las conclusiones sobre el rendimiento de los combustibles, la gasolina súper ofrece un mejor rendimiento en cuanto a recorrido, representando un menor costo a largo plazo. Sin embargo, no se están tomando en cuenta otros parámetros que incurren en costos adicionales, como la facilidad de absorción de agua por parte del etanol presente en el combustible Ecopaís, representa un daño a largo plazo a las partes metálicas que puedan sufrir corrosión, así como la absorción de impurezas junto con la humedad presente en el combustible.

Palabras Claves: consumo de combustible; motor de combustión interna; costo de combustible; rendimiento vehicular; pruebas vehiculares

Abstract: This study is based on an objective, systematic, and controlled investigation performed with the purpose of analyzing the performance, cost, and emissions testing between Super and Ecopais fuels, using a 2017 Chevrolet Aveo model, 1500cc engine, four-cylinder vehicle. The study performs a comparative analysis of four predetermined routes, circulation schedules under similar working conditions for the vehicle. In the methodological aspect, literature related to the topic was reviewed to obtain appropriate data regarding the performance of the two types of fuels available in Ecuador and the use of support applications available online like Google Maps, GPS Speedometer, and Hudspeed were also employed. Using these digital devices like route trackers, allowed performing different performance tests to determine if both fuel types fulfilled the vehicle's parameters for consumption and emission levels. This will allow to establish whether the costs of fuel consumption and long-term maintenance for internal combustion engine vehicles are truly reduced. Results regarding fuel consumption are shown in the conclusion, the Super fuel 
offers better consumption mileage, producing a lower long-term cost. However, other parameters that can cause additional costs are not being taken into consideration, like the possibility of water absorption by the ethanol present in the Ecopaís fuel which might pose a long-term damage to the metallic parts suffering corrosion, as well as the absorption of impurities alongside the moisture present in the fuel.

Key Words: fuel consumption; internal combustion engine; fuel cost; vehicle performance; vehicle testing

\section{Introducción}

El combustible que se va a usar siempre queda a consideración del consumidor, sin embargo, no resta importancia comprobar, si en realidad existen efectos negativos en cuanto a rendimiento, economía y en los resultados en las emisiones de los diferentes tipos de combustibles disponibles en Ecuador. Mediante este proyecto se realiza un estudio de las diferencias en el rendimiento de los combustibles tipo Súper y Ecopaís para demostrar las diferencias de estos combustibles, relacionando el costo con el recorrido en kilómetros por galón y la frecuencia de mantenimientos que se deben realizar, respecto al uso de alguno de los dos tipos de gasolina. En una reciente investigación (España, 2015), ante el encarecimiento de las gasolinas y el fin de los subsidios, vuelve al debate con qué combustible alimentar al vehículo para ahorrarse unos dólares. Claro que, por evitar pagar más no hay que arriesgar y dañar el automóvil. Ese es el debate: Qué gasolina es mejor y si debemos pagar más por la Súper. Lo que manifiestan los técnicos en el área es que, tal y como se comercializan la Ecopaís y la Súper, no hay grandes diferencias mecánicas. La primera es una mezcla de gasolina con $5 \%$ de etanol anhidro, la segunda es gasolina $100 \%$, de 90 octanos, el profesor e investigador de la Universidad Politécnica del Litoral, Juan Peralta, afirma que la nomenclatura y bibliografía internacional fijan hasta entre un 10 y un $15 \%$ el nivel de mezcla (alcohol-gasolina) en el que el motor no tiene variaciones significativas de funcionamiento. Es decir, que con los niveles de mezcla que hay en Ecuador, los componentes de un motor de combustión interna no deberían verse afectados. La misma tesis mantiene el jefe de servicio técnico de Nissan. Como mecánico dice que no tiene evidencia alguna de que el uso de un combustible u otro pueda provocar mayor deterioro de los vehículos. (El comercio, 2017). Este estudio se puede corroborar con datos obtenidos de diversas fuentes de investigación científica como se muestra en la figura 1 el despacho de combustible automotor Ecopaís. 


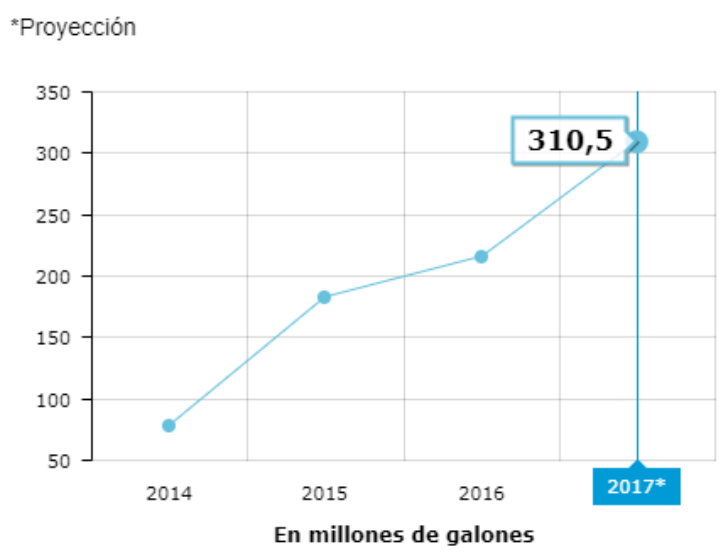

Figura 1. Despacho de combustible automotor Ecopaís (Astudillo \& Pacheco, 2017)

En la figura 2 se puede apreciar la composición del combustible Ecopaís, donde el $60 \%$ es nafta de alto octano, $35 \%$ nafta de bajo octano y el $5 \%$ es bioetanol.

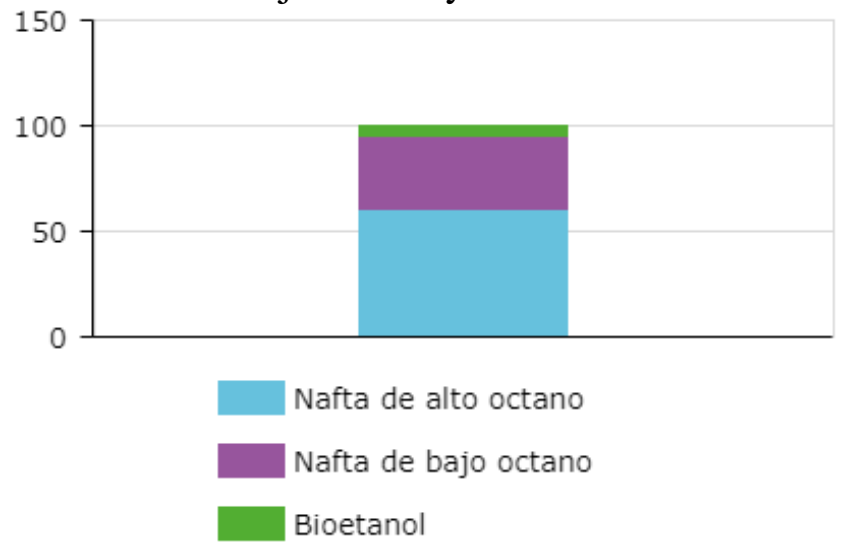

Figura 2. Composición del combustible Ecopaís (Astudillo \& Pacheco, 2017).

En la figura 3 se puede apreciar el despacho de combustible en millones de galones por producto en el año 2017.

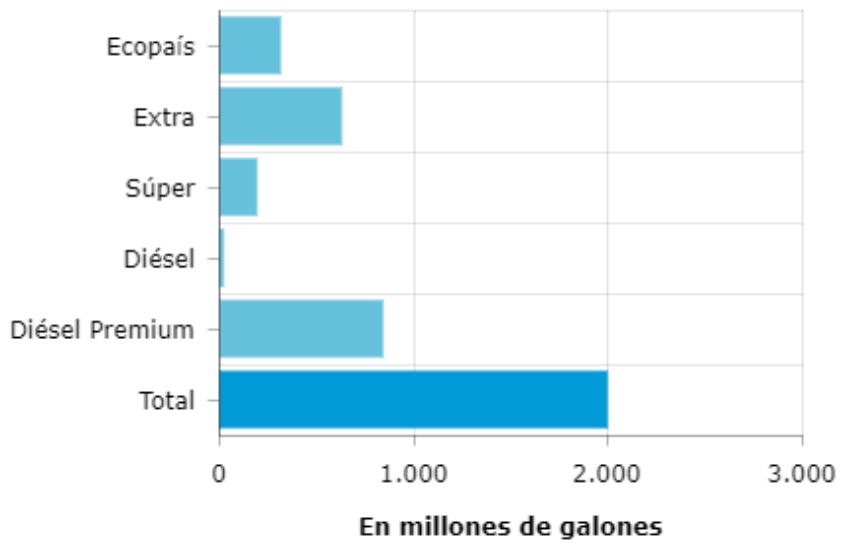

Figura 3. Despacho de combustible por producto en 2017 (Astudillo \& Pacheco, 2017)

\section{La elección de la gasolina Súper sobre la Ecopaís}


El octanaje es la capacidad antidetonante de la gasolina cuando se comprime dentro del cilindro del motor, mientras la relación de compresión de un motor es mayor también es mayor su eficiencia y potencia, pero esto también incrementa el peligro de que se produzca el cascabeleo y se reduzca la potencia y eficiencia del motor. Ahí el octanaje controla el problema, el mayor octanaje de las gasolinas se refleja en un aumento de la eficiencia, sobre todo en motores de inyección. El técnico del Laboratorio de Motores de la Facultad de Ingeniería Mecánica de la ESPE, Alberto Naranjo, explica que el primer paso es escuchar con atención el motor una vez que se implemente la variación del octanaje en la gasolina. Advierte que en caso de que el usuario escuche que su motor comienza a "cascabelear", debe entonces tomar medidas, según Naranjo en caso de registrarse ese problema se debe elegir siempre la gasolina Súper en lugar de la Extra y cambiar sus hábitos de conducción. (El comercio, 2017)

Según representantes de la Cámara Nacional de Distribuidores de Derivados de Petróleo, entre el 2012 y el 2013 el consumo de la gasolina extra subió un 6,1\% mientras que el consumo de la gasolina Súper bajó 5,1\% esto se explica por el aumento del octanaje en la gasolina Extra. Naranjo también indica que se pueden usar aditivos para mejorar el octanaje, sin embargo, advierte que este uso no debe ser habitual sino más bien esporádico, porque los aditivos están compuestos de químicos que pueden generar reacciones negativas en los componentes del motor. El consultor automotriz Édison Yánez opina que hay aditivos que limpian el motor y los potencializan adecuadamente, pero advierte que desde enero pasado estos productos han tenido problemas en ingresar al país a causa de la restricción de las importaciones, añade que una variación negativa del octanaje de la gasolina también producirá más residuos en el motor por lo que los mantenimientos pueden volverse más seguidos.

\section{Industria petrolera y el estado ecuatoriano}

La decisión de las autoridades energéticas del país de reemplazar progresivamente la gasolina extra por un combustible más amigable con el medioambiente dependió de la capacidad que tenía la industria para proveer del biocombustible. El 13 de mayo del 2015, se firmó el Decreto Ejecutivo 675, con el que se oficializó la venta en todo el país de la gasolina Ecopaís, con un costo de USD 1,48 el galón.

El decreto es el epílogo de un plan progresivo de sustitución que el gobierno anunció en el 2010, y que tuvo como piloto un grupo de gasolineras de Guayaquil y de otras ciudades con alto movimiento vehicular en la provincia del Guayas. En la composición de esta gasolina denominada ecológica hay un 5\% de bioetanol (alcohol anhidro) que, en el caso de Ecuador, se genera a partir del procesamiento del bagazo (desecho) de la caña de azúcar. El decreto del ejecutivo precisa que el reemplazo se hizo según fue creciendo la oferta del producto orgánico. Por el lado del Gobierno, se estimaba que la sustitución del combustible se traduciría en al menos 9000 nuevos empleos, una inversión privada de USD 470 millones y, por extensión una disminución en el rubro de la importación de naftas.

Ricardo Rivadeneira, presidente ejecutivo del Ingenio Azucarero Valdez, del grupo empresarial Noboa, consideraba que ese proceso para abastecer al país con suficiente alcohol "ya se estaba haciendo". No obstante, el ejecutivo agregó que demanda de una fuerte inversión, no solo en la ampliación de la superficie destinada al cultivo de caña, sino también en la 
construcción de la infraestructura para su procesamiento, principalmente en nuevas destilerías. Valdez procesa el bioetanol como un subproducto de la extracción del azúcar. (Redacción revista líderes , 2015)

Su producción, explica Rivadeneira, es consecuencia del procesamiento de la caña, y en su caso no implica reducir la producción para destinarla a la elaboración de alcohol. Las proyecciones del Gobierno son que alrededor de unas 30000 hectáreas de sembríos de caña se destinen a la producción de bioetanol hasta este año. Para este año se esperaba que la Ecopaís acapare al menos el 45\% del consumo, en marzo del 2014, Petroecuador firmó un contrato de compraventa de 40 millones de litros de bioetanol Soderal, unidad que pertenece al Grupo Inver San Carlos que también lo integra el Ingenio Azucarero San Carlos comprometió la entrega de 9,5 millones de litros. El gerente administrativo, Augusto Ayala señala que actualmente cubren el $31 \%$ de la producción nacional de caña de azúcar, para asegurar un adecuado abastecimiento del bioetanol, el Ministerio de Agricultura y Ganadería puso en marcha el Programa Nacional de Agro energía, que financia los costos de producción del primer año de siembra. El uso de la gasolina Ecopaís, además de representar un ahorro para el estado en importación de gasolinas de alto octano, constituirá una mejora en la eficiencia de los motores, según indican expertos. Estos expresan que, para utilizar este nuevo tipo de gasolina, los usuarios finales no tendrán que realizar ningún tipo de ajuste en los motores o en los vehículos. Dentro de su informe de rendición de cuentas, Petroecuador dio a conocer que hasta octubre del año pasado la oferta de gasolina Ecopaís (con el 5\% de componente de etanol), era de 3000 barriles diarios. Sin embargo, desde el último trimestre, la oferta subió a 10000 barriles y actualmente llega a los 12 000 diarios.

El experto automotriz, Alexis Ortiz, explica que los autos no deben tener ninguna adecuación, siempre que el etanol en la gasolina no supere el 15\% y en el caso de la Ecopaís, actualmente tiene el 5\%. Asegura además que no importa que se mezcle la Súper o la Extra con la Ecopaís, el motor no tendrá ningún tipo de daño. Lo que sí puede ocurrir, asevera Ortiz, es que baje la durabilidad de filtro del motor en un $30 \%$ en el primer cambio de la gasolina, porque el etanol limpia con mayor eficacia las impurezas del motor y ello provocará que se desgaste más el filtro al principio. 


\section{Materiales y métodos}

En este proyecto se basa en una investigación objetiva, sistemática y controlada con el propósito de analizar y examinar los resultados de rendimiento, economía y pruebas de emisiones entre las combustibles seleccionados. Incluye dos variables en cuanto a combustibles disponibles en el mercado del Ecuador en un grupo de prueba según una marca reconocida en el campo automotriz actual, tomando la marca comercial más vendida en el año 2017, siendo el modelo Chevrolet Aveo Family que ha alcanzado gran popularidad entre los consumidores por su versatilidad y economía (Diario Regional Independiente, 2017), como se muestra en la figura 4.

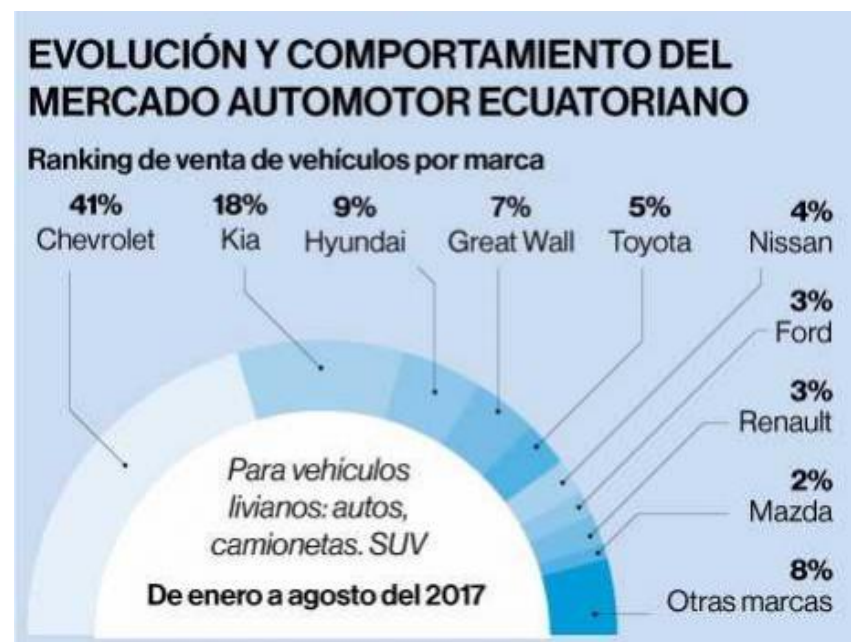

Figura 4. Evolución y comportamiento del mercado automotor ecuatoriano (El comercio, 2017).

Los métodos de análisis que se utiliza fueron aplicaciones de internet que nos demuestran el recorrido total, la velocidad promedio, el tiempo utilizado para realizar el recorrido, con lo que se obtiene datos del consumo de combustible, mediante tablas comparativas. Mediante un método de investigación cuantitativa se ha recopilado información para contrastar las diferencias que producen el uso de los combustibles Súper y Ecopaís, tanto en rendimiento, consumo, emisiones de gases y costos que representan a corto plazo, para demostrar que tipo de combustible es el más adecuado a utilizar, para lo cual se realizan cuatro pruebas de ruta y un análisis de los gases de escape.

\section{Primera prueba mapeo y ruta}

En la primera prueba se realiza un recorrido por una zona de tráfico alto, en un horario de alrededor de 18:00, empezando desde la calle Leopoldo Izquieta Pérez, hasta la Calle 15A NO, dando como resultado un tiempo de recorrido de 16 minutos, en una distancia de 6.5 kilometros como se muestra en la figura 5, logrando una velocidad media de alrededor de $24[\mathrm{~km} / \mathrm{h}]$, mientas se realiza las diferentes pruebas se va utilizar las aplicaciones Google Maps y Hudspeed para registrar el recorrido, la distancia y el tiempo que tomó cumplir la ruta, así como la velocidad promedio y la velocidad máxima de $60[\mathrm{~km} / \mathrm{h}]$. 


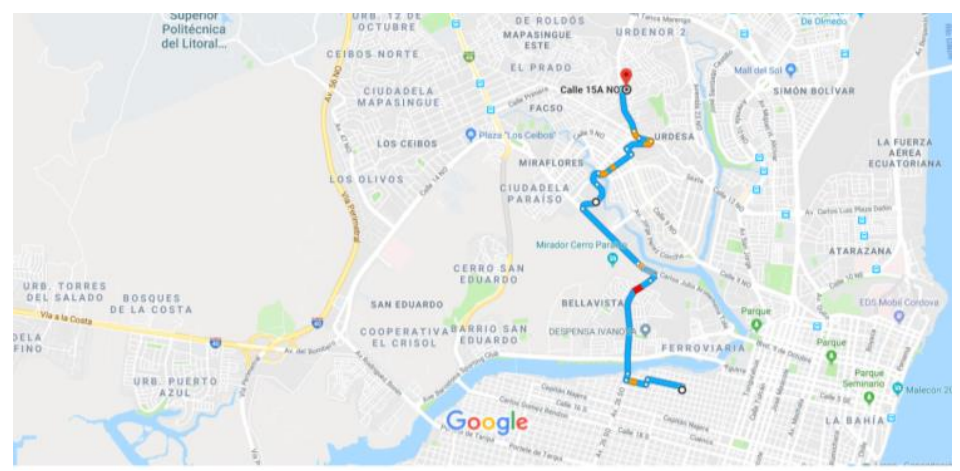

Figura 5. Recorrido en automóvil 6.5 km, 16min (Google Maps, 2017).

\section{Segunda prueba mapeo y ruta}

En la segunda prueba se realiza un recorrido por una zona de tráfico medio, en un horario de alrededor de 8:00, empezando desde la calle Leopoldo Izquieta Pérez, hasta el Fortín, dando como resultado un tiempo de recorrido de 25 minutos, en una distancia de 18.2 kilometros. Logrando una velocidad media de alrededor de $44[\mathrm{~km} / \mathrm{h}]$ y registrando una velocidad maxima de $80[\mathrm{~km} / \mathrm{h}]$, como se muestra en la figura 6.

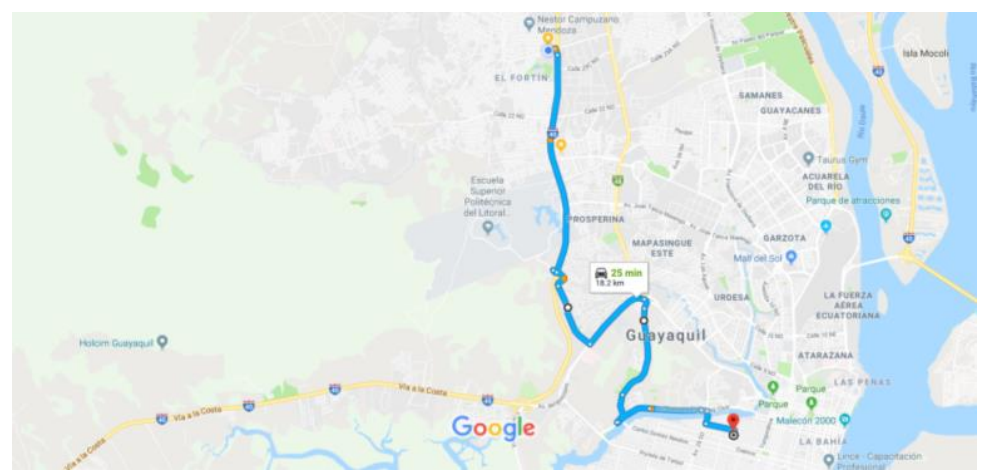

Figura 6. Recorrido en automóvil 18.2km, 25min (Google Maps, 2017).

\section{Tercera prueba mapeo y ruta}

En la tercera prueba se realiza un recorrido por una zona de tráfico bajo, en un horario de alrededor de 14:00, empezando desde el Fortín, hasta la Calle 19 NO en la Florida, dando como resultado un tiempo de recorrido de 9 minutos, en una distancia de 3.6 kilometros. En esta prueba se recorrió a una velocidad media de alrededor de $24[\mathrm{~km} / \mathrm{h}]$ y una velocidad máxima de $100[\mathrm{~km} / \mathrm{h}]$, como se muestra en la figura 7. 


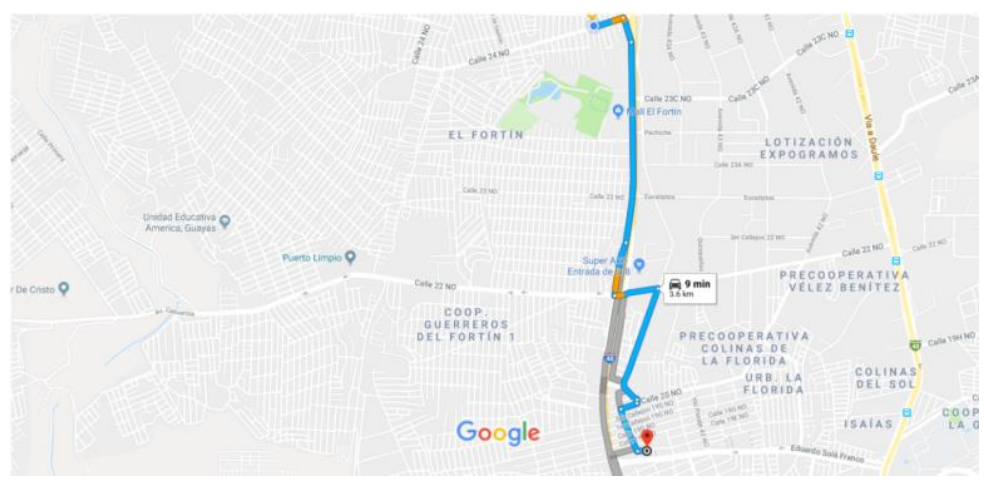

Figura 7. Recorrido en automóvil 3.6km, 9min (Google Maps, 2017).

\section{Cuarta prueba mapeo y ruta}

En la cuarta prueba se realiza un recorrido por una zona de tráfico bajo, en un horario de alrededor de 07:00, empezando desde la calle Leopoldo Izquieta, hasta el zoologico el Pantanal, dando como resultado un tiempo de recorrido de 33 minutos, en una distancia de 23.9

kilometros. Con una velocidad media de alrededor de $44[\mathrm{~km} / \mathrm{h}]$ y una velocidad máxima de 100 $[\mathrm{km} / \mathrm{h}]$, como se ve en la figura 8.

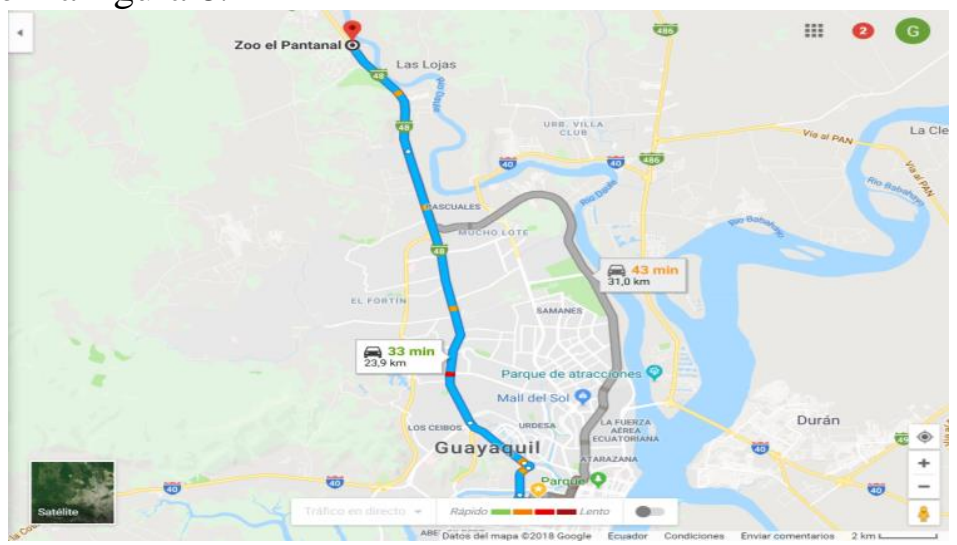

Figura 8. Recorrido en automóvil 23.9km, 33min (Google Maps, 2017).

\section{Gráfica de velocidad de ruta}

Mediante la aplicación de Hudspeed se determina el comportamiento del vehículo en cuanto a velocidades y tiempos de recorridos de rutas, así se logra obtener gráficas de velocidad donde se muestran los picos de las velocidades máximas y mínimas durante el recorrido, además se da un registro real de las paradas y arranques realizados, así como la velocidad promedio de ruta, como se muestra en la figura 9. 


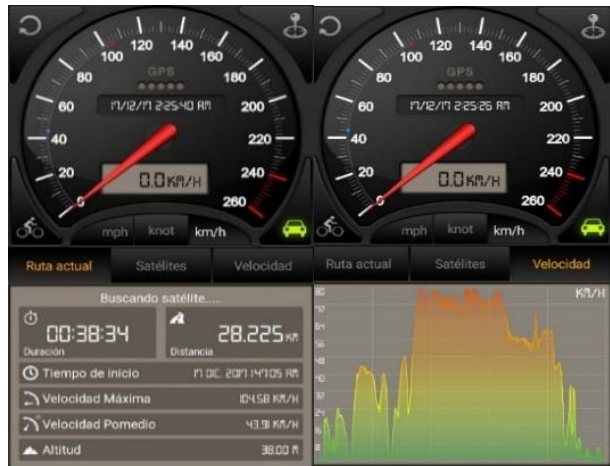

Figura 9. Captura de diagrama de velocidad de ruta (Hudspeed, 2017).

En la tabla 1, registramos los parámetros obtenidos en las diferentes pruebas de ruta y mapeo, calculando el rendimiento de cada galón en kilómetros recorridos. Como se muestra en la figura $10 \mathrm{y}$ en la figura 11 para establecer una relación entre ambos combustibles.

Tabla 1. Comparación gasolina Súper y Ecopaís en un vehículo Chevrolet Aveo Family

\begin{tabular}{|c|c|c|c|c|c|c|c|c|c|c|}
\hline \multirow{3}{*}{ \# } & \multicolumn{2}{|c|}{ Gasolina Súper } & \multirow{3}{*}{$\begin{array}{l}\text { Velocida } \\
\text { d promedio } \\
(\mathrm{km} / \mathrm{h})\end{array}$} & \multirow{3}{*}{${ }_{\mathrm{o}}$ Horari } & \multirow{3}{*}{$\begin{array}{l}\text { Consum } \\
\text { o ciudad } \\
(\mathrm{km} / \mathrm{gal})\end{array}$} & \multicolumn{2}{|c|}{ Gasolina Ecopaís } & \multirow{3}{*}{$\begin{array}{l}\text { Velocida } \\
\text { d promedio } \\
(\mathrm{km} / \mathrm{h})\end{array}$} & \multirow{3}{*}{ o Horari } & \multirow{3}{*}{$\begin{array}{l}\text { Consum } \\
\text { o ciudad } \\
(\mathrm{km} / \mathrm{gal})\end{array}$} \\
\hline & Distanci & Tiemp & & & & Distanci & Tiemp & & & \\
\hline & $\mathrm{a}(\mathrm{km})$ & $\mathrm{o}(\min )$ & & & & $\mathrm{a}(\mathrm{km})$ & $\mathrm{o}(\min )$ & & & \\
\hline 1 & 6.5 & 16 & 24 & 18:00 & 37.5 & 6.5 & 16 & 24 & 18:00 & 36.2 \\
\hline 2 & 18.2 & 25 & 44 & 08:00 & 37.2 & 18.2 & 25 & 44 & 08:00 & 35.9 \\
\hline 3 & 3.6 & 9 & 24 & $02: 00$ & 37.0 & 3.6 & 9 & 24 & 02:00 & 35.7 \\
\hline 4 & 23.9 & 33 & 44 & 07:00 & 37.1 & 28.2 & 33 & 44 & 07:00 & 35.8 \\
\hline
\end{tabular}

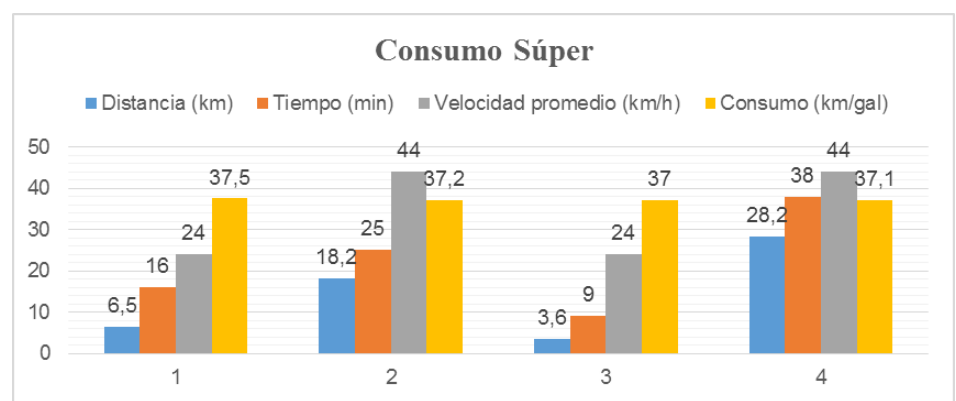

Figura 10. Distancia recorrida y consumo gasolina Súper.

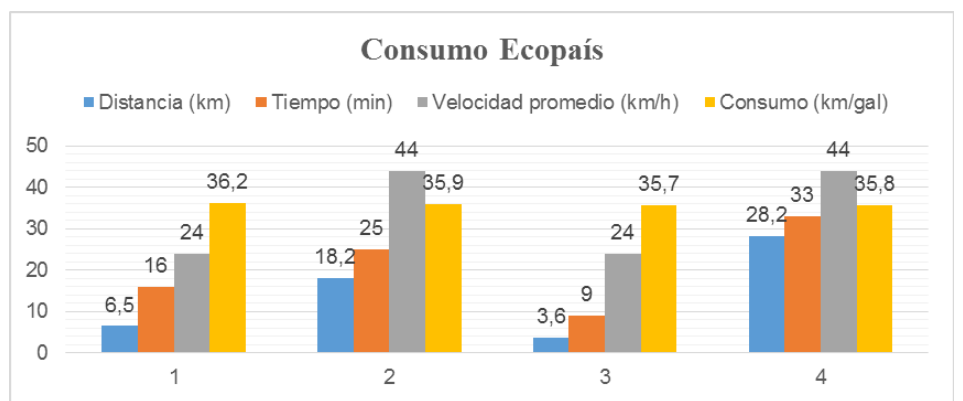

Figura 11. Distancia recorrida y consumo gasolina Ecopaís. 


\section{Analizador de gases}

Al realizar una prueba con el analizador de gases, se registraron las emisiones de los combustibles Súper y Ecopaís; estas pruebas fueron realizadas en la condición de ralentí, es decir, debajo de $1100 \mathrm{rpm}$, donde podemos observar que la gasolina Ecopaís se encuentra muy próximo a los límites permitidos de óxidos nitrosos, así como no observamos una gran diferencia entre los porcentajes de emisiones de monóxido de carbono en ambos combustibles; e incluso, podemos apreciar que el factor de hidrocarburos no quemados y el oxígeno presente aumenta en el uso del combustible Ecopaís, como se muestra en la tabla 2. Además, apreciamos en la figura 12 el comportamiento de los gases de escape.

Tabla 2. Emisiones en combustibles Súper y Ecopaís debajo de 1100 rpm

\begin{tabular}{cccccccc}
\hline & \multicolumn{2}{c}{ Factores en gasolina Súper por galón } & \multicolumn{5}{c}{ Factores en gasolina Ecopaís por galón } \\
NOX\% & CO \% & HC & O2\% & NOX\% & CO\% & HC & O2\% \\
\hline $\mathbf{0 . 5 1}$ & 0.52 & 280 & 3.50 & 0.61 & 0.51 & 286 & 3.80 \\
Límite permitido & & & & & & \\
$\mathbf{0 . 6 2}$ & 1.70 & 350 & 4.00 & 0.62 & 1.70 & 350 & 4.00 \\
\hline
\end{tabular}

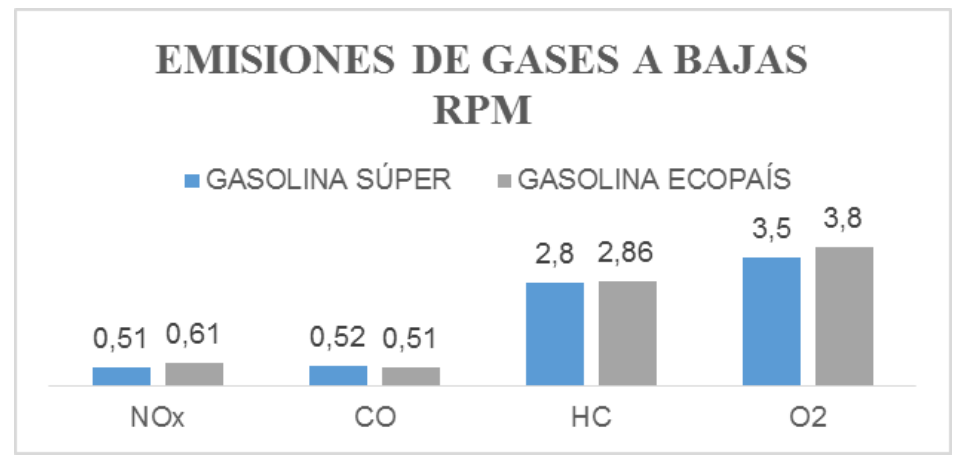

Figura 12. Emisiones de gases a bajas rpm.

También se realiza la prueba de gases de escape en altas revoluciones es decir aproximadamente a $2500 \mathrm{rpm}$, considerando también gases como los hidrocarburos no quemados, el monóxido de carbono, los óxidos nitrosos y el oxígeno presente en los gases de escape, como se muestra en la tabla 3. En esta prueba observamos una disminución entre los porcentajes de emisiones de monóxido de carbono en el combustible Ecopaís; sin embargo, se mantiene la superioridad de los factores de hidrocarburos no quemados y el oxígeno presente es mayor en el uso del combustible Ecopaís, además apreciamos en la figura 13 el comportamiento de los gases de escape.

Tabla 3. Emisiones en combustibles, Súper y Ecopaís aproximadamente a 2500 rpm

\begin{tabular}{cccccccc}
\hline \multicolumn{4}{c}{ Factores en gasolina Súper por galón } & \multicolumn{5}{c}{ Factores en gasolina Ecopaís por galón } \\
NOX\% & CO \% & HC & O2\% & NOX\% & CO\% & HC & O2\% \\
\hline $\mathbf{0 . 5 9}$ & 0.51 & 220 & 3.05 & 0.62 & 0.49 & 226 & 3.26 \\
$\begin{array}{c}\text { Límite permitido } \\
\mathbf{0 . 6 2}\end{array}$ & 1.70 & 350 & 4.00 & 0.62 & 1.70 & 350 & 4.00 \\
\hline
\end{tabular}




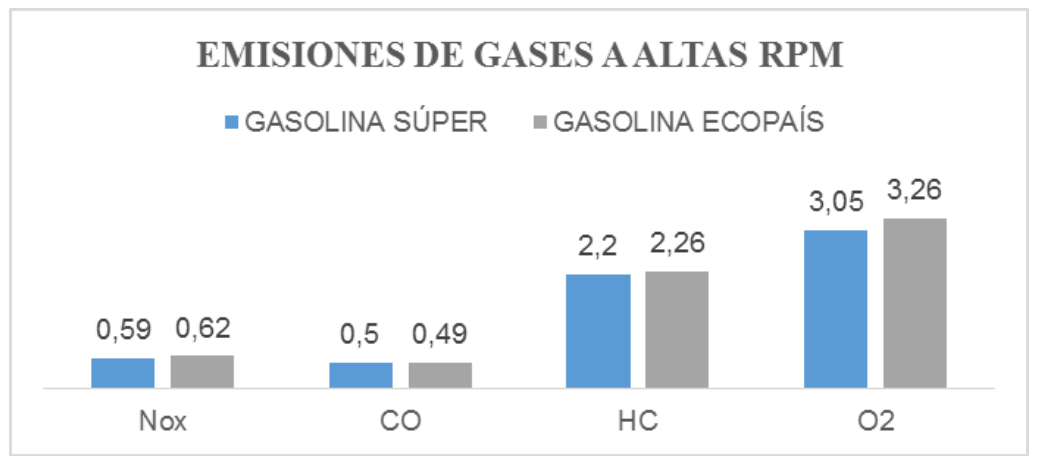

Figura 13. Emisiones de gases a altas rpm.

\section{Análisis de resultados}

En la figura 14 podemos observar que el rendimiento del combustible Súper es superior al rendimiento por galón que nos representa el combustible Ecopaís, lo cual a la larga representa un costo mayor si se toman en cuenta recorridos largos, especialmente en sectores donde se encuentra mucho tráfico, ya que la Ecopaís inclusive en el desempeño deja mucho que desear por experiencia del conductor asignado en esta investigación, quien manifestó que se siente una pérdida de potencia en el arranque al usar el combustible Ecopaís.

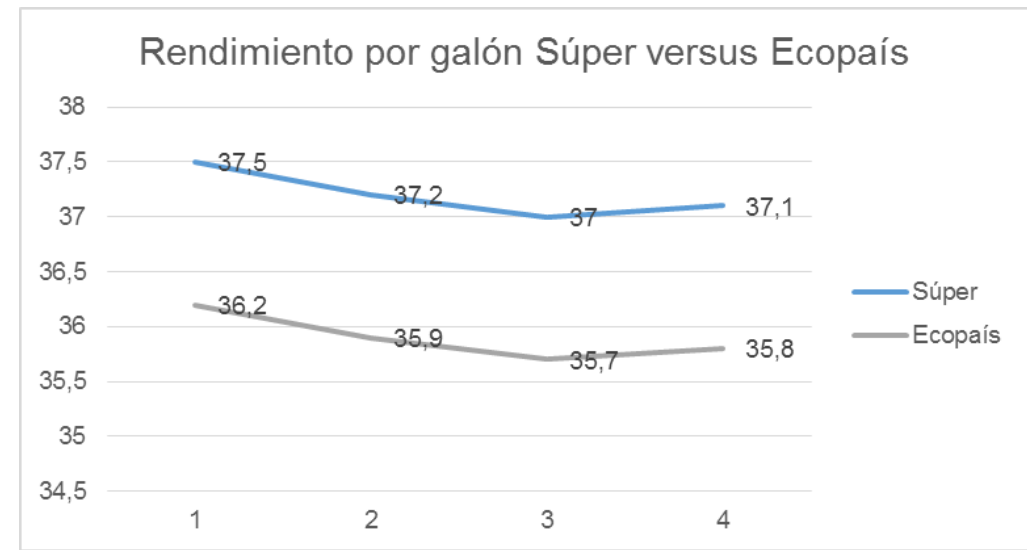

Figura 14. Comparativa del rendimiento Súper versus Ecopaís (km/gal).

Si comparamos los precios de los combustibles Súper y Ecopaís a primera instancia puede parecer que se logra un gran ahorro al utilizar el de menor costo, como se muestra en la tabla 4, sin embargo no se toma en cuenta otros parámetros que incurren en costos adicionales como lo demostramos en la figura 15 de rendimiento por galón de cada combustible, además de que la facilidad de absorción de agua por parte del etanol presente en el combustible Ecopaís representa un daño a largo plazo a las partes metálicas que puedan sufrir corrosión así como la absorción de impurezas junto con la humedad presente en el combustible antes mencionado.

Tabla 4. Precio oficial de gasolinas Súper y Ecopaís (Astudillo \& Pacheco, 2017)

\begin{tabular}{llll}
\hline \multicolumn{2}{c}{ Gasolina Súper por galón } & \multicolumn{2}{c}{ Gasolina Ecopaís por galón } \\
Año & Costo en dólares & Año & Costo en dólares \\
\hline 2016 & 2.05 & 2016 & 1.42 \\
2017 & 2.98 & 2017 & 1.47 \\
\hline
\end{tabular}




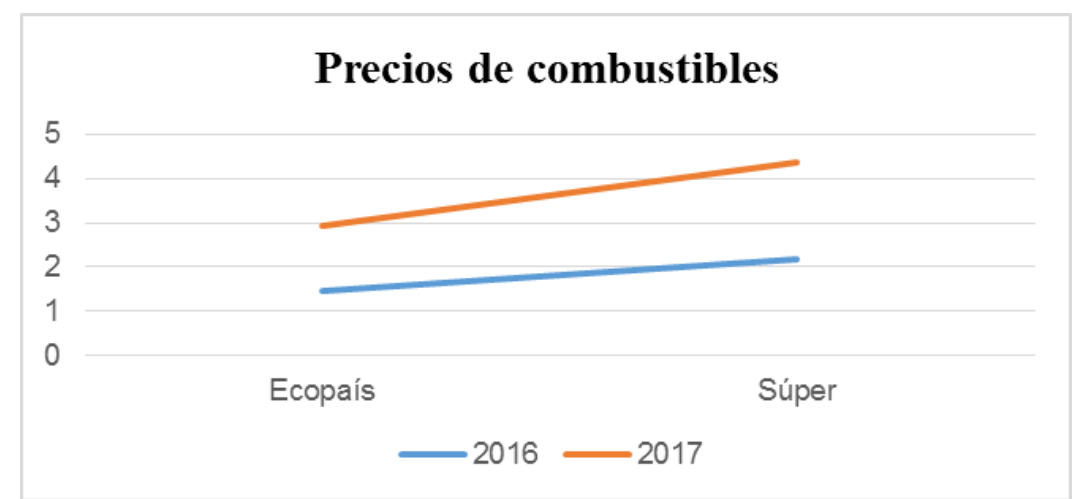

Figura 15. Precio oficial de combustibles (Astudillo \& Pacheco, 2017).

En la tabla 5 se muestra el consumo en galones que se utilizó en cada prueba, obteniendo como resultado en el mismo recorrido un costo mayor en todos los casos puesto que la cantidad en volumen compensa el precio del combustible más económico, en la figura 16 y en la figura 17 donde podemos observar gráficas similares, dándonos una idea del consumo y los costos que representa usar cada tipo de combustible.

Tabla 5. Consumo de gasolina versus costos

\begin{tabular}{lllllll}
\hline \multicolumn{4}{l}{ Gasolina Súper por galón } & \multicolumn{3}{c}{ Gasolina Ecopaís por galón } \\
\# & Galones & Precio & Total & Galones & Precio & Total \\
\hline $\mathbf{1}$ & 0,17 & 2.98 & 0,50 & 0,18 & 1.47 & 0,40 \\
$\mathbf{2}$ & 0,10 & 2.98 & 0,29 & 0,10 & 1.47 & 0,22 \\
$\mathbf{3}$ & 0,49 & 2.98 & 1,46 & 0,51 & 1.47 & 1,12 \\
$\mathbf{4}$ & 0,76 & 2.98 & 2,26 & 0,79 & 1.47 & 1,73 \\
\hline
\end{tabular}

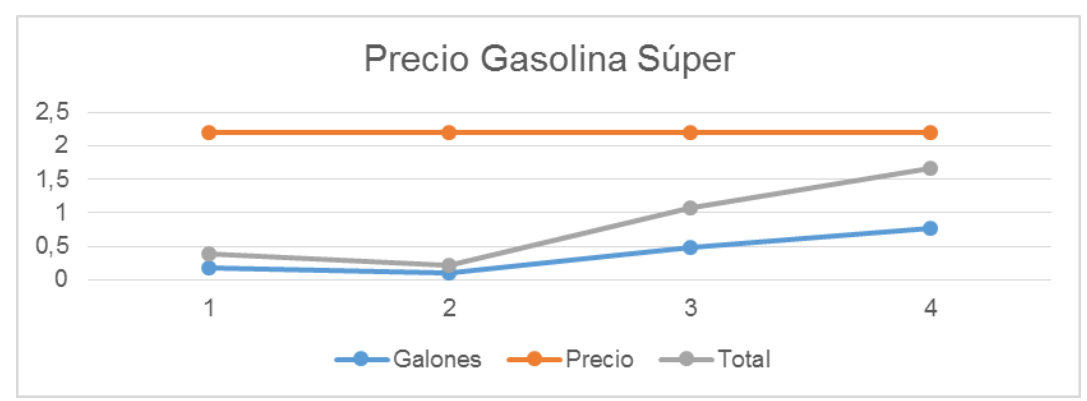

Figura 16. Consumo de gasolina Súper.

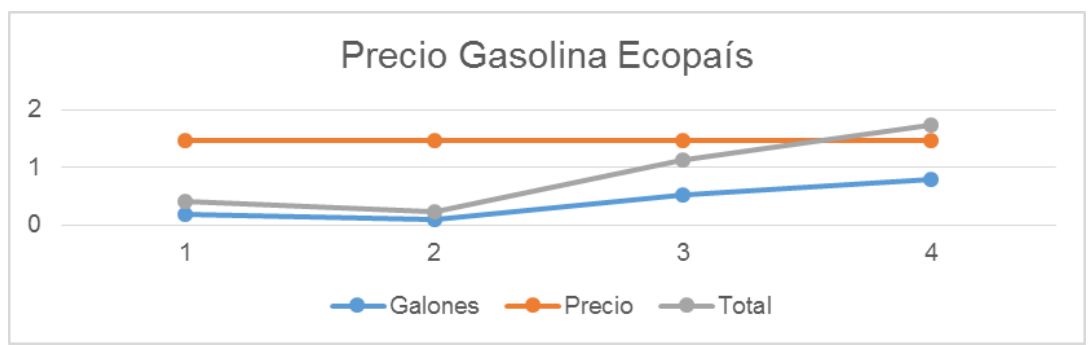

Figura 17. Consumo de gasolina Ecopaís. 
En la figura 18, observamos una comparativa de las emisiones de los combustibles Súper y Ecopaís; estas pruebas fueron realizadas en la condición de altas revoluciones, y en ralentí. En esta prueba se observa, que en ambas condiciones el combustible Ecopaís se encuentra sobre los factores de emisiones del combustible Súper, sin cambios significativos en el monóxido de carbono, así como en los óxidos nitrosos que se encuentran en el límite con el uso de Ecopaís; manteniendo la superioridad de los factores de hidrocarburos no quemados y el oxígeno presente es mayor en el uso de este combustible, por lo cual no podemos asegurar que exista un beneficio ecológico representativo.

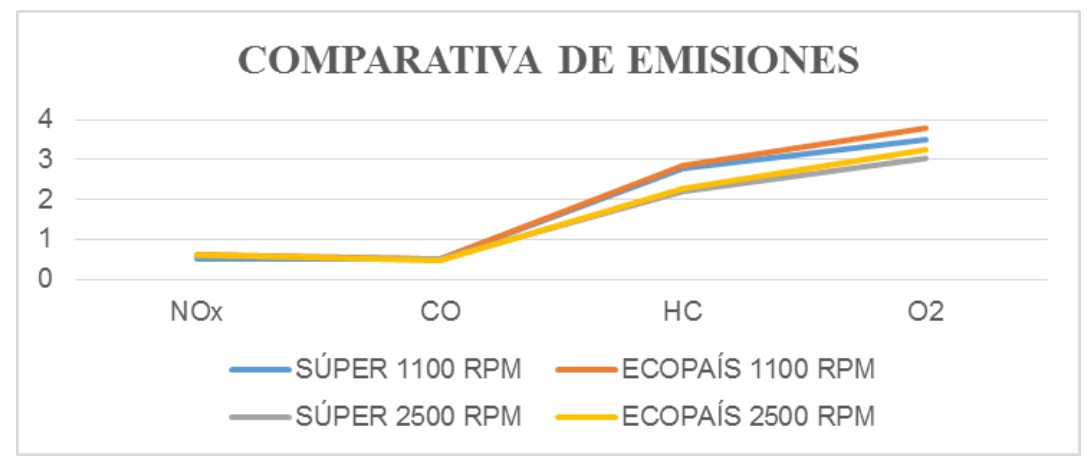

Figura 18. Emisiones de gases comparativas

\section{Discusiones finales}

- Se observó que la gasolina Súper ofrece un mejor rendimiento en cuanto a recorrido, representando un menor costo a largo plazo.

- Se compararon las características en cuanto a la percepción del conductor, percibiendo el mismo, bajo desarrollo del motor, así como, pérdidas de potencia en el arranque, con el uso del combustible Ecopaís.

- Las emisiones de gases resultantes nos demuestran que el combustible súper genera un \% menor de gases contaminantes que el combustible Ecopaís.

- La gasolina Ecopaís al tener un $5 \%$ de etanol el cual representa un oxigenante para el combustible, por lo que la mezcla de aire combustible va a tener una mayor cantidad de oxígeno, esto se ve representado en el porcentaje de emisiones de contaminantes como el CO, según los datos obtenidos de las pruebas se encuentra en un porcentaje menor en relación con el combustible Super.

- Los vehículos de alta gama necesitan un combustible de mejor calidad y al momento de cambiar por un combustible de un octanaje menor, el vehículo tendrá problemas y empezará a cascabelear, lo que no sucede con los vehículos de gama media que pueden cambiar sin problema, aunque la potencia se verá afectada como la vida útil de ciertos componentes del motor.

\section{Bibliografía}

Acelerando transporte. (2015). Acelerando. Obtenido de Transporte: http://www.acelerando.com.ec/industria/mundo-verde/443-gasolina-eco-paisreemplazara-a-extra-hasta-el-2017 
Alvarez Flórez, J. A., \& Callejón Agramunt, I. (2004). Maquinas térmicas motoras (volumen I). Catalunya: Edicions UPC.

Araujo, A. (2015). Cómo actuar ante la baja de octanaje de la gasolina. Obtenido de El Comercio: http://www.elcomercio.com/actualidad/negocios/actuar-baja-de-octanaje-de.html.

Astudillo, G., \& Pacheco, M. (25 de Agosto de 2017). La Ecopaís está disponible en el 40\% de las estaciones. Obtenido de El comercio.

Bravo, V. (17 de Julio de 2006). Supermotor. Obtenido de Revista noticias hyundai accent: http://www.supermotor.com/revista/2006/07/267911.html

Chavez, R. A., Hoyo, L. R., \& Esquivel , O. (2015). XXVI Congreso De Investigación CUAM AC Mor. México: Centro Universitario Anglo Mexicano.

Chevrolet. (2017). Aveo Family. Obtenido de http://www.chevrolet.com.ec/aveo-family-autoeconomico.html

Diario Regional Independiente. (2017). El Norte. Obtenido de http://www.elnorte.ec/economia/27055-el-top-5-de-los-autos-m\%C3\%A1s-vendidos-enecuador.html

El comercio. (21 de Octubre de 2017). Marcas lanzaron 30 nuevos modelos de autos en el 2017. Obtenido de http://www.elcomercio.com/actualidad/venta-autos-ecuador-negocioseconomia.html

El telégrafo. (6 de Mayo de 2017). El telégrafo. Obtenido de El precio de los combustibles en Ecuador es el más bajo de la región: http://www.eltelegrafo.com.ec/noticias/economia/8/el-precio-de-los-combustibles-enecuador-es-el-mas-bajo-de-la-region

España, S. (23 de octubre de 2015). La súper gana a la mezcla. Obtenido de Expreso: http://www.espol.edu.ec/espol/docs_escribe/3905.pdf

Gallardo, R. (2015). Materiales de un motor de combustión interna. . Obtenido de http://www.academia.edu/8812101/Materiales_De_Un_Motor_De_Combustion_Interna

INEN. (2017). Revenido, NTE INEN 0671. Obtenido de Recubrimientos electrolíticos de zinc sobre acero y requisitos: www.inen.gov.ec

Redacción revista líderes . (31 de mayo de 2015). El mercado se adecúa a la gasolina Ecopaís. Obtenido de Revista líderes: http://www.revistalideres.ec/lideres/mercado-gasolinaecopais-ecuador.html.

Sánchez Pescador, J. (Noviembre de 2015). Grado en Ingeniería Mecánica. Simulación dinámica de mecanismos con Autodesk Inventor Professional 2014. Valladolid. Obtenido de Simulación dinámica de mecanismos con Autodesk Inventor Professional 2014. 
Sempere, J. (2014). Escola professional salesianos. Obtenido de Motor Wankel: http://sva.salesianos.edu/alcoy.juanxxiii/sites/salesianos.edu.alcoy.juanxxiii/files/sites/def ault/files/motor_wankel.pdf 